\title{
Kernel-Based Learning-Aided Phase Noise Compensation in Dual-Pump Optical Phase Conjugation Coherent System
}

\author{
T. T. Nguyen ${ }^{1, \dagger}$, S. Boscolo ${ }^{1, *}$, A. A. I. Ali ${ }^{1}$, M. Tan ${ }^{1}$, T. Zhang ${ }^{1}$, S. Takasaka ${ }^{2}$, R. \\ Sugizaki $^{2}$, S. Sygletos ${ }^{1}$, and A. D. Ellis ${ }^{1}$ \\ ${ }^{1}$ Aston Institute of Photonic Technologies, Aston University, Birmingham, B4 7ET, United Kingdom \\ ${ }^{2}$ Furukawa Electric Co. Ltd., 6 Yawata-kaigandori, Ichihara, Chiba, 290-8555, Japan \\ ${ }^{\dagger}$ Now with Infinera PA, 7360 Windsor Dr, Allentown, PA 18106, USA. *s.a.boscolo@aston.ac.uk
}

\begin{abstract}
We deploy kernel-based time-series prediction to suppress the phase noise induced by small deviations from ideal pump counter-phasing in a dual-pump optical phase conjugation system. We show experimentally $1.5-\mathrm{dB}$ SNR improvement for 16-QAM signals at $4^{\circ}$ pumpphase mismatch. (C) 2021 The Author(s)
\end{abstract}

\section{Introduction}

Optical phase conjugation (OPC) has been shown as a promising method to mitigate the distortions caused by chromatic dispersion and Kerr nonlinearities in high-transmission rate dispersion-unmanaged coherent optical systems through mid-span spectral inversion [1,2]. However, a major limitation to the performance of fibre-based OPC devices relying on four-wave mixing is the transfer of the pump phase modulation that is used to suppress stimulated Brillouin scattering from the pump to the conjugated signal (idler). Theoretically, this transfer can be fully suppressed by the use of a dual-pump scheme in which the two pumps are modulated using complementary phase patterns [3]. However, this approach requires precise adjustment of the phase and amplitude of the modulating signals, which is difficult to achieve in practice. Therefore, small deviations from the ideal case commonly exist, and require the development of advanced digital signal processing (DSP) to counteract their side effects.

In this paper, we propose for the first time the aid of machine learning (ML) to conventional DSP phase-noise (PN) compensation [4] to correct the deterministic phase distortion caused by deviations from ideal pump counter-dithering in the OPC of quadrature-amplitude modulation (QAM) signals. We deploy a kernel-based recursive least-squares (KRLS) time-series prediction method [5] in which the phase of the signal after conventional PN compensation is regarded as the result of a time-varying process. The online algorithm then tracks these time variations by considering data in windows of fixed size and calculating the updated solution for each window. Contrary to existing compensation methods [6], the proposed approach requires no prior knowledge of the dithering frequencies and features high sensitivity to the residual pump dithering. The latter characteristic makes it particularly suitable for offsetting small imperfections in the pump-phase modulation scheme of OPC-assisted systems. The technique is verified experimentally with a 28 Gbaud dual-polarisation 16-QAM signal, and shown to achieve $1.5 \mathrm{~dB}$ signal-to-noise ratio (SNR) improvement relative to unaided PN compensation at a pump-phase mismatch of $4^{\circ}$ within an optical back-to-back OPC configuration.

\section{Experimental Setup}

The experimental setup of the dual-polarisation 28-Gbaud 16-QAM system that was used to validate the proposed PN compensation method is shown in Fig. 1. At the transmitter, 64000 random 16-QAM symbols were generated for each polarisation. The data was then up-sampled at 2 samples per symbol and pulse-shaped using a root-raised-cosine filter with a 0.1 roll-off factor. The signal was loaded into a Keysight arbitrary waveform generator (AWG) (4-channel 8-bit digital-to-analogue converter with 56-GSa/s sample rate) and subsequently converted into the optical domain by a commercial multi-format optical transmitter ( $\sim 100-\mathrm{kHz}$ laser linewidth on 192.4-THz). An erbium-doped fibre amplifier was used to control the launch signal power.

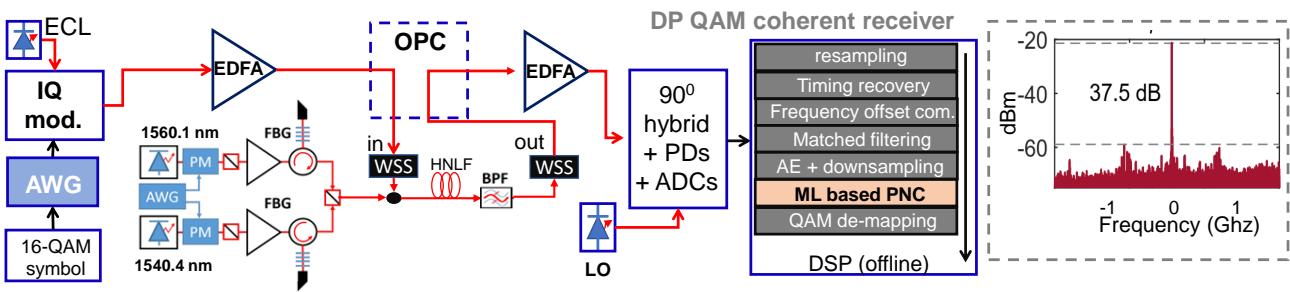

Fig. 1. Experimental setup of the OPC-assisted dual-polarisation 28 Gbaud 16-QAM system equipped with ML-assisted PN compensation.

The signal was conjugated through a polarisation-insensitive dual-band OPC with orthogonally polarised pumps spectrally located at $1540.4 \mathrm{~nm}$ and $1560.1 \mathrm{~nm}$, and with laser linewidth $\sim 30 \mathrm{kHz}$. The details of the OPC setup can be found in [2]. One signal band only was used in this experiment. Two radio-frequency (RF) tones at frequencies $f_{1}=$ $60 \mathrm{MHz}$ and $f_{2}=600 \mathrm{MHz}$ were generated by the AWG and used to independently phase-modulate the pump lasers via 
optical phase modulators. The amplitudes and phases $\theta_{i}$ of the RF tones for each phase modulator $i=1,2$, were first adjusted to minimise the transfer of phase modulation from the pumps to the generated idler. The inset of Fig. 1 shows the RF spectrum of the photo-detected conjugated copy of a continuous-wave laser after the OPC in these calibration settings. It features $37.5 \mathrm{~dB}$ suppression (relative to the carrier peak) of the phase-modulation sidebands transferred from the pumps. We refer to this operating condition as ideal pump counter-phasing, which we can express as $\delta \theta=$ $\left(\theta_{1}+\theta_{2}\right)-180^{\circ}=0^{\circ}$, assuming a fully compensated modulation-index mismatch between the two modulators. Then, we intentionally increased the phase mismatch $\delta \theta$ up to $8^{\circ}$ by tuning the phase of one of the RF tones. The HNLF was $100 \mathrm{~m}$ long, and the nominal values of the fibre attenuation coefficient, Kerr-nonlinearity coefficient, zero-dispersion wavelength, and dispersion slope were $1.2 \mathrm{~dB} \mathrm{~km}^{-1}, 21.4 \mathrm{~W}^{-1} \mathrm{~km}^{-1}, 1550 \mathrm{~nm}$, and $0.041 \mathrm{ps} \mathrm{nm}^{-2} \mathrm{~km}^{-1}$, respectively.

Detection of the conjugated copy of the signal was performed with a typical intra-dyne coherent receiver including a local oscillator $(\sim 100-\mathrm{kHz}$ linewidth on $193.1 \mathrm{THz})$, a $90^{\circ}$ optical hybrid, four balanced photo-detectors and a realtime sampling scope (100-GS/s, $23 \mathrm{GHz}$ analogue bandwidth). A standard DSP procedure for data recovery [7,8] was implemented offline. The DSP started with re-sampling the digital signal at 2 samples per symbol. Timing recovery and frequency-offset error correction were performed using a Gardner phase detector (window size of 1024) and a conventional Fourier-transform-based method (window size of 4096), respectively. After matched filtering, an adaptive equaliser (15-tap butterfly-structure) demultiplexed the dual-polarisation fields and compensated linear effects. The signal was then down-sampled to 1 sample per symbol before being fed into the proposed PN compensation module. At the output of this module, the recovered QAM symbols together with the transmitted symbols were used to measure an effective SNR defined as $\mathrm{SNR}=\mathbb{E}_{k}\left[|x[k]|^{2}\right] / \mathbb{E}_{k}\left[|\hat{y}[k]-x[k]|^{2}\right]$, where $x[k]$ and $\hat{y}[k]$ are the respective transmitted and received QAM symbols at the time instance $k$ and $\mathbb{E}$ is the expectation operator.

\section{Kernel-Based Learning-Assisted PN Compensation Method}

Assuming for simplicity perfect timing recovery, ideal synchronisation and zero frequency-offset, the phase-distorted signal at the input to the PN compensation unit can be written in the form $y[k]=x[k] \exp \left[i\left(\delta \phi[k]+\phi_{m}[k]\right)\right]+\varepsilon[k]$, where $\varepsilon[k]$ is the additive white Gaussian noise in the system. The first phase term $\phi[k] \sim \mathscr{N}\left(0,2 \pi \delta v T_{S}\right)$ represents the Wiener random laser PN, where $\delta v$ is the combined spectral linewidth of the system (total linewidth of transmit, receive and pump lasers) and $T_{s}$ is the symbol period. The second phase term $\phi_{m}[k]=\phi_{m 1}[k]+\phi_{m 2}[k] \sim$ $(m+\delta m)\left[\cos \left(2 \pi f_{1} t_{k}+\delta \theta\right)+\cos \left(2 \pi f_{2} t_{k}+\delta \theta\right)\right]+m\left[-\cos \left(2 \pi f_{1} t_{k}\right)-\cos \left(2 \pi f_{2} t_{k}\right)\right]$ represents the deterministic phase distortion generated by imperfect pump counter-phasing, where $m$ is the modulation index and the modulation-index mismatch $\delta m$ represents possibly different modulation indices. Note that in practice, this phase term contains also nonlinear mixing products arising from the nonlinear response of the RF amplifiers. In an OPC-free coherent system, the well-known blind-phase-search (BPS) method [4] is the preferred scheme to compensate for laser-induced PN for the 16-QAM format because it does not require the use of pilot symbols. However, the effectiveness of this method may degrade significantly under the impact of the PN term arising in an OPC system because of its decision-directed operation. To circumvent this issue, we deploy an additional ML-based compensation stage to aid the BPS algorithm, as depicted in Fig. 2.

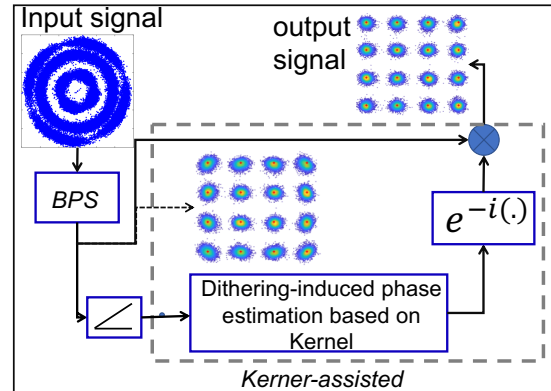

(a)

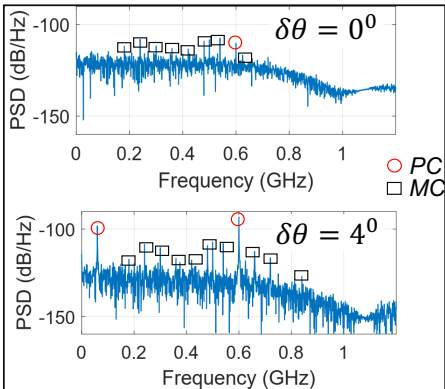

(b)

Fig. 2. (a) Block diagram of the proposed PN compensation scheme. (b) Spectral representation of the estimated phase noise after the ML unit. PC: Principal components (i.e., the RF frequencies), MC: mixing components.

Hence, within the proposed scheme, the PN-distorted signal is first phase-compensated by the BPS algorithm (using 32 test phase angles and a filter width of 7). We denote here the output signal from the BPS stage as $\hat{\boldsymbol{Y}}_{1}$. Then the signal's phase is stripped off and fed into the ML unit whose operational principle relies upon the slidingwindow KRLS algorithm presented in [5]. Slightly differently from [5], our implementation of the algorithm seeks the optimal model vector $\boldsymbol{h}$ that solves the LS problem: $\min _{\boldsymbol{h}}\left\|\angle\left\{\left[\hat{\boldsymbol{Y}}_{1} \boldsymbol{h}\right\rfloor_{\mathrm{D}}\right\}-\angle\left\{\hat{\boldsymbol{Y}}_{\mathbf{1}} \boldsymbol{h}\right\}\right\|^{2}$, where $\angle\{$.$\} and \lfloor\cdot\rfloor_{\mathrm{D}}$ are the angle and direct-decision operators, respectively. The remainder of the implementation follows Algorithm 1 in [5], where we use a window size $N$, regularisation parameter $c$ and Gaussian kernel width $w$ of 26, 0.5 and 100, respectively. The estimated evolution of the phase is then used to compensate for the remaining phase distortion after the BPS block (Fig. 2(a)). Fig. 2(b) shows examples of the estimated PN after the ML unit in the frequency domain for the pump-phase mismatches $\delta \theta=0^{\circ}$ and $4^{\circ}$ and $\sim 36 \mathrm{~dB}$ optical SNR. It is noteworthy that even in the "ideal" pump 
counter-phasing configuration, there are still several frequency components transferred from the RF tones and their linear and nonlinear mixing to the idler, indicating the persistence of some residual dithering. These components can be detected by the proposed ML-assisted scheme. We also observe that the larger $\delta \theta$ the higher the amplitude of these components.

\section{Results and Discussion}

To evaluate the effectiveness of the proposed PN compensation scheme, we set the OPC transceiver to operate at an optical SNR of $\sim 36 \mathrm{~dB}$ under ideal pump counter-phasing $\left(\delta \theta=0^{\circ}\right)$. Then we assess the performance of PN compensation in terms of effective SNR when varying the pump-phase mismatch $\delta \theta$. The results are summarised in Fig. 3. We observe that the performance of the conventional BPS method degrades significantly under the impact of imperfect pump counter-phasing: more than 1-dB SNR penalty is observed at a pump-phase mismatch of only $4^{\circ}$. Conversely, the ML-aided scheme gives around $0.3 \mathrm{~dB}$ performance benefit over conventional BPS under the best pump-dithering settings (i.e., at $\delta \theta=0^{\circ}$ ), and this is not lost until the pump-phase mismatch is $4^{\circ}$. At $\delta \theta=4^{\circ}$, the ML-assisted BPS method outperforms conventional BPS by $1.5 \mathrm{~dB}$, with this value increasing to $3 \mathrm{~dB}$ when $\delta \theta$ reaches $8^{\circ}$.

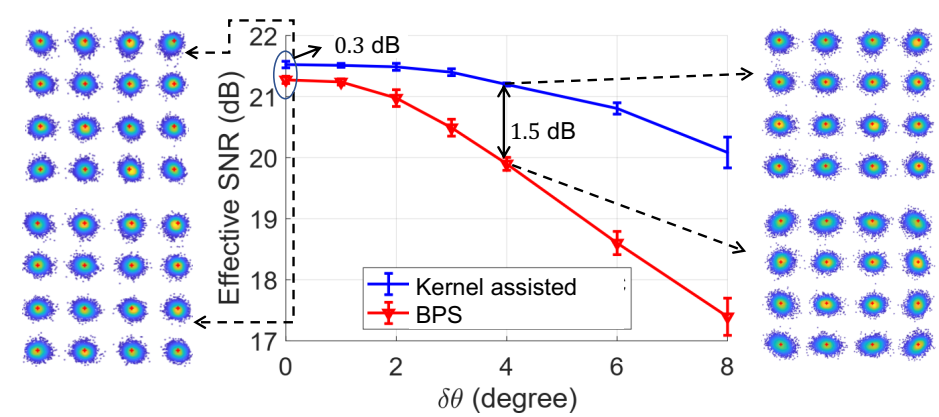

Fig. 3. Performance comparison between KRLS-assisted and conventional PN compensation in terms of effective SNR versus pump-phase mismatch $\delta \theta$ for 16-QAM system.

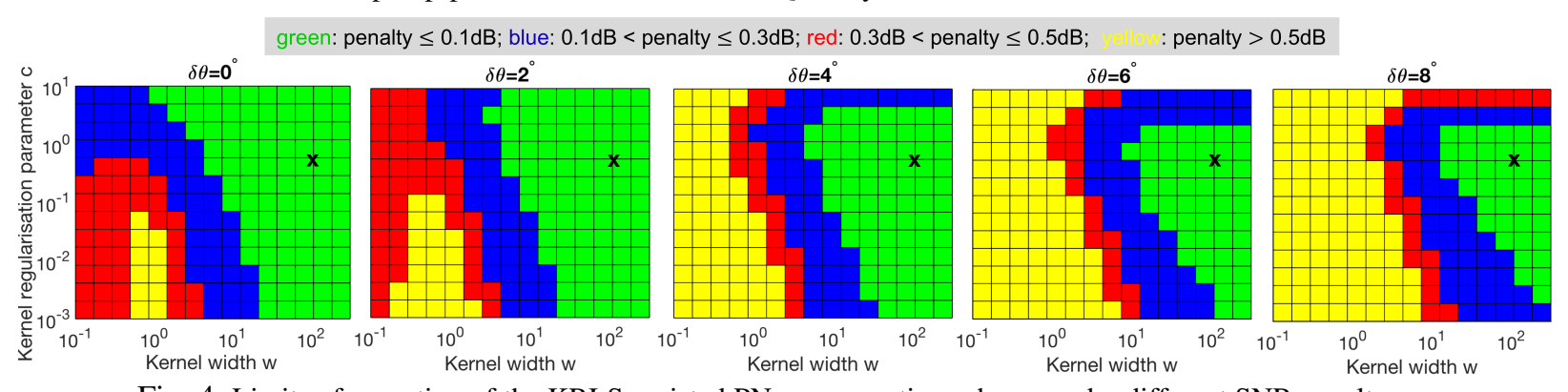

Fig. 4. Limits of operation of the KRLS-assisted PN compensation scheme under different SNR penalty constraints in the plane of kernel regularisation parameter $c$ and kernel width $w$ for $\delta \theta=0^{\circ}, 2^{\circ}, 4^{\circ}, 6^{\circ}$, and $8^{\circ}$. The $\mathrm{X}$ marks indicate the parameter set $(c, w)$ used in Fig. 3.

Execution of KRLS algorithm requires knowledge of the kernel's parameters which are not available analytically. In Fig. 4, we shows the working ranges of kernel's parameter values within different SNR penalty constraints for different pump-phase mismatch values. For each $\delta \theta$ value we calculate the SNR penalty with respect to the maximum effective SNR obtained at that phase mismatch as $\max \left\{\forall \mathrm{SNR}_{c, w}\right\}-\mathrm{SNR}_{c, w}$, where $\mathrm{SNR}_{c, w}$ is the $\mathrm{SNR}$ at a given parameter set $(c, w)$. The results in Fig. 4 highlight that there is considerable flexibility in the choice of $c$ and $w$ subject to minor performance degradation. This stems from the adaptive operation of the kernel method. We can see in particular that there is a large overlap between the parameter regions bearing a SNR penalty of $\leq 0.1 \mathrm{~dB}$ (green areas) for the tested $\delta \theta$ values. This means that the same set $(c, w)$ can be chosen to feed the PN compensation scheme for different pump-phase mismatch levels.

\section{Conclusion}

We have developed a KRLS algorithm-enhanced PN compensation method able to estimate and correct the phase distortion arising from small imperfections in the pump-modulation scheme of OPC-assisted coherent systems, which commonly exist in practice even in optimised OPC setups. By applying the technique to a 16-QAM system, we have demonstrated 1.5-dB SNR improvement relative to conventional PN compensation at a pump-phase mismatch of $4^{\circ}$. Owing to its non-parametric nature, the deployed KRLS algorithm is indifferent to the number of dithering frequencies used, which makes our method potentially applicable to cascaded OPC systems.

Acknowledgements: This work was supported by the UK EPSRC - Grants EP/S003436/1, EP/S016171/1 and EP/R035342/1.

\section{References}

1. A. D. Ellis et al., Adv. Opt. Photon. 9, 429-503 (2017).

2. M. A. Z. Al-Khateeb et al., Opt. Express 26, 23960-23969 (2018).

3. M.-C. Ho et al., J. Lightwave Technol. 20, 469-476 (2002).
4. T. Pfau et al., J. Lightwave Technol. 27, 989-999 (2009).

5. S. Van Vaerenbergh et al., Proc. IEEE ICASSP 2006, pp. V-V.

6. R. Elschner et al., Proc. ECOC 2010, P3.17.

7. S. J. Savory, Opt. Express 16, 804-817 (2008).

8. T. T. Nguyen et al., J. Lightw. Technol. 39, 388-399 (2021). 